\title{
Amygdala-Hippocampus Dynamic Interaction in Relation to Memory
}

\author{
Gal Richter-Levin* and Irit Akirav \\ Department of Psychology, Laboratory of Behavioral Neuroscience, \\ Haifa University, Haifa 31905, Israel
}

\begin{abstract}
Typically the term "memory" refers to the ability to consciously remember past experiences or previously learned information. This kind of memory is considered to be dependent upon the hippocampal system. However, our emotional state seems to considerably affect the way in which we retain information and the accuracy with which the retention occurs. The amygdala is the most notably involved brain structure in emotional responses and the formation of emotional memories.

In this review we describe a system, composed of the amygdala and the hippocampus, that acts synergistically to form long-term memories of significantly emotional events. These brain structures are activated following an emotional event and cross-talk with each other in the process of consolidation. This dual activation of the amygdala and the hippocampus and the dynamics between them may be what gives emotionally based memories their uniqueness.
\end{abstract}

Index Entries: Amygdala; hippocampus; learning; emotion; stress.

\section{Introduction}

Alterations in brain function in response to experience are presumed to underlie an organism's ability to learn and remember. Cellular, molecular, morphological, and psychological principles have been proposed as underlying

\footnotetext{
*Author to whom all correspondence and reprint requests should be addressed. E-mail: gal.r-1@psy. haifa.ac.il
}

the way in which organisms receive, conduct, transmit, and store information (1).

A vast amount of research has focused on elucidating the role of the hippocampus in learning and memory. The hippocampus is involved in the storage of explicit/declarative memory, often described as the conscious recall of facts and events (2-5). Animal studies have reported complementary observations that the hippocampus is necessary for generating spatio-temporal and contextual representations $(4,6-11)$. 
Memory is not a passive process in which we indiscriminately retain information from our environment. Instead, variables such as context and prior experiences filter which information is retained and the accuracy with which that retention occurs. One important such filter is the emotional state (12).

From an evolutionary and functional perspective, animals (including humans) would appear to benefit little from having memories for trivial events that are as strong as memories for important events. Hence, brain mechanisms have evolved for storing information that reflects the degree to which the information is worth storing (13). An essential component of the neural circuitry underlying emotional responses is the amygdala (14). Initial studies in this field reported that damage to the amygdala decreased the emotional responsivity of animals $(15,16)$. Subsequent work confirmed the essential role of the amygdala in the expression of emotions and the formation of emotion-related memories $(14,17,18)$. Damage to the amygdala in humans and animals results in a profound impairment in learning ability, especially in those tasks that require the subject to make a connection between environmental stimuli and strong emotional responses (17,19-22).

Emotional states range from positive to negative, and both states can range from low to high levels of arousal. Most research has focused on the negative emotional state due to the profound neurobiological effects of stress on learning and memory. These effects are believed to be the basis of many cognitive and affective changes in health and disease (12).

One of the enigmatic findings in the literature is that heightened emotionality can either impair or enhance memory, depending on experimental conditions. Specifically, it was found that the effects of stress and glucocorticoids on hippocampal plasticity and on memory consolidation of hippocampal-dependent learning follow an inverted-U-shape doseresponse relationship: extreme low and high levels may impair plasticity or consolidation (23-30) whereas moderate activation of gluco- corticoids receptors seems to be a prerequisite for the long-term storage of information (31-35).

The aim of the following review is to try to describe a system, focusing on the amygdala and the hippocampus, that acts synergistically to form long-term memories of significantly emotional events. These brain structures are activated following an emotional event and cross-talk with each other in the process of consolidation. This dual activation of the amygdala and the hippocampus and the dynamics between them may be what gives emotionally based memories their uniqueness.

\section{Stress and the Hippocampus}

All living organisms experience stressful events during their lifetime. Stressful stimuli elicit a spectrum of physiological responses vital for the maintenance of homeostasis, the most prominent of which is the activation of the hypothalamic-pituitary-adrenal (HPA) axis.

Brief periods of stress can potentiate memory formation, whereas more severe or prolonged stress can have deleterious effects upon broad aspects of cognition (12). It has been suggested that the effects of stress and in particular glucocorticoids on memory are mediated through their influence on the hippocampus.

One of the best-characterized effects is the capacity of psychological stress and stress induced levels of glucocorticoids to disrupt long-term potentiation (LTP) and primed-burst potentiation (PBP). Numerous of studies, using a broad range of stressors (novelty, restraint, shock, predator exposure) have consistently shown that stress or elevated levels of corticosterone inhibit the induction of excitatory plasticity (LTP and PBP) and promote the induction of inhibitory plasticity (long-term depression; LTD) $(27,29,30,36-45)$. Nevertheless, lower concentrations of corticosterone, such as those that occur naturally during the diurnal rise, enhance such plasticity $(37,46,47)$. 
There is also evidence for the biphasic effects of stress and corticosterone on the performance in hippocampal-dependent forms of learning and memory. There are several studies supporting the positive or permissive role of glucocorticoids in the construction of memories. For example, adrenalectomy or injecting a glucocorticoid receptor (GR) antagonist impairs performance in a spatial learning task (31). Likewise, an intra-cerebral injection of corticosterone enhanced the development of a chick's longterm memory and this effect was reduced by injection of either mineralocorticoid receptor or GR antagonists $(34,48-49)$. Finally, rats injected with the GR antagonist $1 \mathrm{~h}$ prior to or immediately after contextual-fear conditioning displayed less contextual-fear conditioning than rats injected with vehicle (32).

Other studies have shown that acute stressors impair hippocampal-specific learning. For example, inducing a foot shock or corticosterone administration 30 -min before a retention test for the spatial task in the water maze impaired retention (33). In addition, a high dose of corticosterone or GR agonist $2 \mathrm{~h}$ prior to training in the $\mathrm{Y}$ maze resulted in impaired spatial memory (24). Finally, placing rats in close proximity to a cat, which evokes an intense fear response in the rats, impaired their memory for recently acquired spatial information $(26,50)$.

In conclusion, hippocampal functioning is highly sensitive to levels of emotionality. Evidently, stress modulates the ability of the hippocampus to store and retrieve information in an inverted U-shaped function.

\section{Amygdala-Hippocampus Interactions}

Long-term memories are considered to be consolidated over time. Substantial evidence suggests that the slow consolidation of memories serves an adaptive function by enabling endogenous processes activated by an experience to modulate memory strength (51). Adrenal stress hormones, epinephrine, and glucocorticoids released by emotional arousal appear to play an important role in enabling the significance of an experience to regulate the strength of memory of the experience $(30,37,40)$. Activation of the amygdala is critical for mediating the influences of epinephrine and glucocorticoids because amygdala lesions block the effects of these modulators on consolidation (51-53).

The hypothesis that the amygdala modulates hippocampal memory storage is rooted in several lines of research, at the anatomical, electrophysiological, and functional levels.

\section{Anatomical Evidence}

The most widespread projections from the amygdala to the hippocampal formation originate in the basal nucleus, which projects substantially to the entorhinal cortex (EC), CA3 and CA1 fields of the hippocampus, the subiculum, and the parasubiculum. The accessory basal-nucleus projects substantially to three components of the hippocampal formation: the EC, the CA1 field, and the parasubiculum. The main projections from the lateral nucleus are directed to the EC and the parasubiculum (54).

\section{Electrophysiological Evidence}

Evidence from several studies indicates that amygdala activity influences the induction of hippocampal LTP. Population spike (PS)-LTP in the dentate gyrus was attenuated by lesion of the basolateral amygdala (BLA) (55) or by injection of the local anaesthetic tetracaine into the $\operatorname{BLA}(55,56)$.

High-frequency stimulation (HFS) of the basomedial amygdala or the BLA applied at the same time as tetanic stimulation of the perforant path (PP) facilitated dentate gyrus PS-LTP $(57,58)$, whereas lesioning the fimbria-fornix impaired BLA induced reinforcement of LTP in the dentate gyrus (59).

Recently, we found that priming the BLA 30$s$ prior to PP stimulation resulted in the facilitation of the excitatory post-synaptic potentials (EPSP)-LTP in the dentate gyrus (60). 
It should be noted that these effects are not necessarily indicative of a direct, monosynaptic BLA-DG projection. For example, similar consequences could be the result of the activation of the anatomically described projection of the BLA to the DG via a pathway relaying in the EC.

\section{Functional Evidence}

Several lines of evidence support the notion that the amygdala has a modulatory influence on memory-related processes in the hippocampus, although the mechanism of action is not well-understood.

Lesions of the stria terminalis (a major afferent/efferent pathway of the amygdala) were found to attenuate the effects of post-training intra-amygdala drug treatments on hippocampus-dependent memory tasks $(61,62)$. Another study has shown that an intra-amygdala injection of N-methyl-D-aspartate (NMDA) induces $c$-fos expression in the dorsal hippocampus (63).

At the behavioral level, post-training injections of amphetamine into the caudate selectively enhanced retention of a cued task, whereas amphetamine injected into the hippocampus selectively enhanced retention of a spatial task. In contrast, amphetamine injected into the amygdala enhanced retention of both tasks (64).

BLA lesions were also found to have an effect on hippocampal-dependent learning. BLA lesions block the memory-modulating effects induced by a GR agonist infused into the hippocampus (65) and also block memory impairment induced by either adrenalectomy or intrahippocampal infusions of a GR antagonist $(66,67)$.

We have recently shown (36) that priming the amygdala prior to stimulation of the PP modulates hippocampal synaptic plasticity in an interval-dependent manner, i.e., enhancement with a 30-s interval and inhibition with a $1-h$ interval. Furthermore, exposing rats to a stressor 1-h prior to priming the amygdala in proximity to PP stimulation blocked the enhancing effects of BLA priming on hippocampal plasticity.

Based on these results, we proposed that the amygdala has a bi-phasic effect on hippocampal plasticity: a fast excitatory phase that may serve as a marker for emotional events (emotional tagging), and a slower inhibitory phase that may be beneficial in reducing masking effects of following, less-significant events during the initial consolidation stage.

\section{Plasticity in the Amygdala}

The amygdala is an essential component of the neural circuitry involved in emotional responses, in general, and in attaching emotional significance to learned stimuli (14). The specific role of the amygdala in memory formation has been a topic of recent debate $(68,69)$.

While there is an agreement regarding the role of the amygdala in modulating memorystorage processes occurring in other brain regions, there is a dispute over whether or not the amygdala serves as the site of long-term emotional-memory storage (reviewed in ref. 70).

Several lines of evidence support the role of the amygdala in the storage of emotional memories. Post-training lesion studies indicate that the amygdala holds information relevant to some aspects of fear conditioning (71-73), though others seem to be independent of amygdalar contribution $(74,75)$. Both subcortical and cortical inputs to the amygdala were shown to be amenable to LTP (76-78). Furthermore, amygdala cells develop learninginduced changes in discharge activity. These alterations were found to reflect a combination of plasticity projected on the amygdala from its afferents interacting with plasticity intrinsic to the amygdala $(76,79)$. Perhaps the most significant support comes from recent experiments in which fear conditioning was found to induce LTP in the amygdala, both in vivo and in vitro $(80,81)$. Importantly, LTP was not induced by either the conditioned stimulus or the uncon- 
ditioned stimulus alone. Only the conditioned association between the two was effective in inducing LTP in the amygdala. Similarly, we have recently observed activation of MAPK/ERK (mitogen-activated protein kinase/extracellular regulated-kinase) in the amygdala following a stressful spatial-learning paradigm (82). Spatial learning under less stressful conditions or the mere exposure to high levels of stress were not effective, suggesting that the amygdala is involved in storing information relating to the interaction between the emotional and cognitive aspects of the experience. MAPK/ERK activation is likely to activate a cascade of molecular events that will lead to the synthesis of new proteins and the consolidation of long-term memory. Thus, the stress/spatial learning-specific activation of the kinase supports the notion that plasticity within the amygdala subserves some aspects of long-term emotionally charged memories.

Clearly, if a role of the stored aversive events in the amygdala is to enhance memory consolidation in other brain areas that represent and store different aspects of the experience (69), then plasticity within the amygdala can be expected also to modify the way the amygdala exerts this function.

\section{Hippocampus-Amygdala Interactions}

Anatomical studies have revealed that a prominent source of BLA afferents is the hippocampal formation. These projections course through the ventral angular bundle (VAB) and arise from the CA1, the ventral subiculum, and the EC (83-86). Intracellular-recording studies have suggested that hippocampal formationBLA projections are monosynaptic (87) and glutamatergic (88). The hippocampal formation-BLA projection is suspected of having a specific role in Pavlovian fear conditioning, of conveying information about contextual stimuli from the hippocampal formation to the BLA. Maren and Fanselow (77) found that HFS to the VAB produced an enduring LTP. Furthermore, they showed that electrolytic lesions placed in the regions of the hippocampal formation that project to the BLA eliminated Pavlovian fear conditioning to a contextualconditioning stimulus. It has been suggested (89) that the hippocampus contributes to fear conditioning in transmitting complex kinds of signals (e.g., the association of context with the US).

These results indicate to changes in neural responses in the BLA following different hippocampal-induced manipulations. However, further research is necessary for displaying hippocampal formation modulation of amygdala-dependent learning.

\section{Summary}

There has been extraordinary progress in the last few years in trying to understand the neurobiology of learning, particularly learning about emotional events. Emotional events are, of course, accompanied by strong arousal, the mechanisms of which are suggested to promote brain processes responsible for the encoding of new information.

Two main brain areas that are essentially involved in the encoding and consolidation of emotionally charged experiences are the hippocampus and the amygdala. These two brain areas are obviously amenable to changes following an emotional learning experience. The information about an emotional event may be stored in parallel in both structures while each structure probably encodes a different aspect of the experience. Moreover, it has been demonstrated that the amygdala modifies the hippocampus responses and vice versa. Consequently, a dynamic interaction may exist between the amygdala and the hippocampus and this may underlie the dynamic nature of memories. For example, the hippocampus may receive information from the amygdala concerning odors and dangerous stimuli, and from neural circuits involved in classical conditioning. Correspondingly, the amygdala may receive information from the hippocampus that enables it to put the event into the proper con- 
text. Furthermore, experience-dependent alterations in the hippocampal formation will influence the way the amygdala will process future emotional experiences and vise versa. The consolidation of new memories into long-term memories is time dependent and it may be that the cross-talk between the two structures plays an important role in regulating the strength of memory for emotionally based experiences.

The amygdala, the hippocampus, and the stress hormones are key players in emotional learning, but other brain regions and systems are involved as well. For example, the prefrontal cortex was found to actively modulate extinction of amygdala-related aspects of memory $(90,91)$. Stimulating the hippocampus may induce LTP in the prefrontal cortex (92-95) and the amygdala modulates plasticity in the hippocampus $(36,55-57)$. Whether the amygdala directly modulates plasticity in the prefrontal cortex is yet to be studied.

The understanding of the nature of these dynamic connections may shed light on how emotional memories endure and under what circumstances emotion interferes with consolidation. In addition to the understanding of what is the underlying mechanism in normal life situations, it is important to understand what happens in memories that are out of the "ordinary" scale of remembering. Two examples of such extraordinary memories are the flash-bulb memories and flashbacks within post-traumatic stress disorder (PTSD), in which the events were enormously emotional at the time they occurred.

With the understanding gained from the research into the dynamic interactions between relevant brain structures, we can hope for better comprehension of the neural mechanisms involved in affective disorders such as anxiety and PTSD.

\section{References}

1. Rahman H., Freeman S. J., and Rahman M. (1992) The Neurobiological Basis of Memory and Behavior. Springer-Verlag, New York, NY.
2. Eichenbaum H. (1999) The hippocampus and mechanisms of declarative memory. Behav. Brain Res. 103, 123-133.

3. Maguire E. A., Burgess N., and O'Keefe J. (1999) Human spatial navigation: cognitive maps, sexual dimorphism, and neural substrates. Curr. Opin. Neurobiol. 9, 171-177.

4. Squire L. R. (1992) Memory and the hippocampus: a synthesis from findings with rats, monkeys, and humans. Psychol. Rev. 99, 195-231.

5. Squire L. R. (1998) Memory systems. C.R. Acad. Sci. III. 321, 153-156.

6. Alvarez P., Zola-Morgan S., and Squire L. R. (1995) Damage limited to the hippocampal region produces long-lasting memory impairment in monkeys. J. Neurosci. 15, 3796-3807.

7. Bunsey M. and Eichenbaum H. (1996) Conservation of hippocampal memory function in rats and humans. Nature 18, 255-257.

8. Eichenbaum H. (1992) The hippocampal formation and declarative memory in animals. J. Cog. Neurosci. 4, 217-231.

9. Morris R. G., Garrud P., Rawlins J. N., and O'Keefe J. (1982) Place navigation impaired in rats with hippocampal lesions. Nature 24, 681-683.

10. Moser E., Moser M. B., and Andersen P. (1993) Spatial learning impairment parallels the magnitude of dorsal hippocampal lesions, but is hardly present following ventral lesions. J. Neurosci. 13, 3916-3925.

11. O'Keefe J. (1990) A computational theory of the hippocampal cognitive map. Prog. Brain Res. 83, 301-312.

12. McEwen B. S. and Sapolsky R. M. (1995) Stress and cognitive function. Curr. Opin. Neurobiol. 5, 205-216.

13. Cahill L. and McGaugh J. L. (1996) Modulation of memory storage. Curr. Opin. Neurobiol. 6, 237-242.

14. LeDoux J. E. (1993) Emotional memory systems in the brain. Behav. Brain Res. 20, 69-79.

15. Kluver H. and Bucy P. (1937) "Psychic blindness" and other symptoms following bilateral temporal lobectomy in rhesus monkeys. Am. J. Physiol. 119, 352-353.

16. Weiskrantz L. (1956) Behavioral changes associated with ablation of the amygdaloid complex in monkeys. J. Comp. Physiol. Psychol. 49, 381-391.

17. Davis M. (1992) The role of the amygdala in fear-potentiated startle: implications for animal models of anxiety. TIPS 13, 35-41. 
18. McGaugh J. L., Introini-Collison I. B., Nagahara A. H., Cahill L., Brioni J. D., and Castellano C. (1990) Involvement of the amygdaloid complex in neuromodulatory influences on memory storage. Neurosci. Biobehav. Rev. 14, 425-431.

19. Adolphs R., Tranel D., Damasio H., and Damasio A. R. (1995) Fear and the human amygdala. J. Neurosci. 15, 5879-5891.

20. Davis M. (1994) The role of the amygdala in emotional learning. Int. Rev. Neurobiol. 36, 225-266.

21. LaBar K. S., LeDoux J. E., Spencer D. D., and Phelps E. A. (1995) Impaired fear conditioning following unilateral temporal lobectomy in humans. J. Neurosci. 15, 6846-6855.

22. Maren S., Aharonov G., and Fanselow M. S. (1996) Retrograde abolition of conditional fear after excitotoxic lesions in the basolateral amygdala of rats: absence of a temporal gradient. Behav. Neurosci. 110, 718-726.

23. Conrad C. D., Galea L. A. M., Kuroda Y., and McEwen B. S. (1996) Chronic stress impairs rat spatial memory on the $Y$ maze, and this effect is blocked by tianeptine pretreatment. Behav. Neurosci. 6, 1321-1334.

24. Conrad C. D., Lupien S. J., and McEwen B. S. (1999) Support for a bimodal role for type 2 adrenal steroid receptors in spatial memory. Neurobiol. Learn. Mem. 72, 39-46.

25. Diamond D. M., Fleshner M., and Rose G. M. (1994) Psychological stress repeatedly blocks hippocampal primed burst potentiation in behaving rats. Behav. Brain Res. 30, 1-9.

26. Diamond D. M., Park C. R., Heman K. L., and Rose G. M. (1999) Exposing rats to a predator impairs spatial working memory in the radial arm water maze. Hippocampus 9, 542-552.

27. Diamond D. M. and Rose G. M. (1994) Stress impairs LTP and hippocampal-dependent memory. Ann. NY Acad. Sci. 746, 411-414.

28. Luine V., Villegas M., Martinez C., and McEwen B. S. (1994) Repeated stress causes reversible impairments of spatial memory performance. Brain Res. 639, 167-170.

29. Pavlides C., Ogawa S., Kimura A., and McEwen B. S. (1996) Role of adrenal steroid mineralocorticoid and glucocorticoid receptors in long-term potentiation in the CA1 field of hippocampal slices. Brain Res. 4, 229-235.

30. Pavlides C., Watanabe Y., and McEwen B. S. (1993) Effects of glucocorticoids on hippocampal long-term potentiation. Hippocampus 3, 183-192.
31. Oitzl M. S. and de Kloet E. R. (1992) Selective corticosteroid antagonists modulate specific aspects of spatial orientation learning. Behav. Neurosci. 106, 62-71.

32. Pugh C. R., Fleshner M., and Rudy J. W. (1997) Type II glucocorticoid receptor antagonists impair contextual but not auditory-cue fear conditioning in juvenile rats. Neurobiol. Learn. Mem. 67, 75-9.

33. de Quervain D. J., Roozendaal B., and McGaugh J. L. (1998) Stress and glucocorticoids impair retrieval of long-term spatial memory. Nature 20, 787-790.

34. Sandi C. and Rose S. P. R. (1994a) Corticosteroid receptor antagonists are amnestic for passive avoidance learning in day-old chicks. Eur. J. Neurosci. 6, 1292-1297.

35. Sandi C., Loscertales M., and Guaza C. (1997) Experience-dependent facilitating effect of corticosterone on spatial memory formation in the water maze. Eur. J. Neurosci. 9, 637-642.

36. Akirav I. and Richter-Levin G. (1999b) Biphasic modulation of hippocampal plasticity by behavioral stress and basolateral amygdala stimulation in the rat. J. Neurosci. 1, 10,530-10,535.

37. Diamond D. M., Bennett M. C., Fleshner M., and Rose G. M. (1992) Inverted-U relationship between the level of peripheral corticosterone and the magnitude of hippocampal primed burst potentiation. Hippocampus 2, 421-430.

38. Foy M. R., Stanton M. E., Levine S., and Thompson R. F. (1987) Behavioral stress impairs longterm potentiation in rodent hippocampus. Behav. Neural. Biol. 48, 138-149.

39. Garcia R., Musleh W., Tocco G., Thompson R. F., and Baudry M. (1997) Time-dependent blockade of STP and LTP in hippocampal slices following acute stress in mice. Neurosci. Lett. 12, 41-44.

40. Rey M., Carlier E., Talmi M., and SoumireuMourat B. (1994) Corticosterone effects on longterm potentiation in mouse hippocampal slices. Neuroendocrinology 60, 36-41.

41. Shors T. J., Seib T. B., Levine S., and Thompson R. F. (1989) Inescapable versus escapable shock modulates long-term potentiation in the rat hippocampus. Science 14, 224-226.

42. Shors T. J., Gallegos R. A., and Breindl A. (1997) Transient and persistent consequences of acute stress on long-term potentiation (LTP), synaptic efficacy, theta rhythms and bursts in area CA1 of the hippocampus. Synapse 26, 209-217. 
43. Wang J., Akirav I., and Richter-Levin G. (2000) Short-term behavioral and electrophysiological consequences of underwater trauma in the rat. Physiol. Behav. 70, 327-332.

44. Xu L., Anwyl R., and Rowan M. J. (1997) Behavioral stress facilitates the induction of long-term depression in the hippocampus. Nature 29, 497-500.

45. Xu L., Anwyl R., and Rowan M. J. (1998) Spatial exploration induces a persistent reversal of long-term potentiation in rat hippocampus. Nature 27, 891-894.

46. Kerr D. S., Huggett A. M., and Abraham W. C. (1994) Modulation of hippocampal long-term potentiation and long-term depression by corticosteroid receptors activation. Psychobiology 22, 123-133.

47. Pavlides C. Kimura A., Magarinos A. M., and McEwen B. S. (1994) Type I adrenal steroid receptors prolong hippocampal long-term potentiation. Neuroreport 20, 2673-2677.

48. Rose S. P. (1995) Cell-adhesion molecules, glucocorticoids and long-term-memory formation. TINS 18, 502-506.

49. Sandi C. and Rose S. P. R. (1994b) Corticosterone enhances long-term retention in one-day-old chicks trained in a weak passive avoidance learning paradigm. Brain Res. 647, 106-112.

50. Diamond D. M., Fleshner M., Ingersoll N., and Rose G. M. (1996) Psychological stress impairs spatial working memory: relevance to electrophysiological studies of hippocampal function. Behav Neurosci. 110, 661-672.

51. McGaugh J. L. (2000) Memory: a century of consolidation. Science 14, 248-251.

52. Liang K. C., McGaugh J. L., and Yao H. Y. (1990) Involvement of amygdala pathways in the influence of post-training intra-amygdala norepinephrine and peripheral epinephrine on memory storage. Brain Res. 5, 225-233.

53. Roozendaal B. and McGaugh J. L. (1996a) Amygdaloid nuclei lesions differentially affect glucocorticoid-induced memory enhancement in an inhibitory avoidance task. Neurobiol. Learn. Mem. 65, 1-8.

54. Pikkarainen M., Ronkko S., Savander V., Insausti R., and Pitkanen A. (1999) Projections from the lateral, basal, and accessory basal nuclei of the amygdala to the hippocampal formation in rat. J. Comp. Neurol. 11, 229-260.

55. Ikegaya Y., Saito H., and Abe K. (1994) Attenuated hippocampal long-term potentiation in basolateral amygdala-lesioned rats. Brain Res. 5, 157-164.
56. Ikegaya Y., Saito H., and Abe K. (1995) Requirement of basolateral amygdala neuron activity for the induction of long-term potentiation in the dentate gyrus in vivo. Brain Res. 13, 351-354.

57. Ikegaya Y., Abe K., Saito H., and Nishiyama N. (1995) Medial amygdala enhances synaptic transmission and synaptic plasticity in the dentate gyrus of rats in vivo. J. Neurophysiol. 74, 2201-2203.

58. Ikegaya Y., Saito H., and Abe K. (1996) The basomedial and basolateral amygdaloid nuclei contribute to the induction of long-term potentiation in the dentate gyrus in vivo. Eur. J. Neurosci. 8, 1833-1839.

59. Jas J., Almaguer W., Frey J. U., and Bergado J. (2000) Lesioning the fimbriafornix impairs basolateral amygdala induced reinforcement of LTP in the dentate gyrus. Brain Res. 7, 186-189.

60. Akirav I. and Richter-Levin G. (1999a) Priming stimulation in the basolateral amygdala modulates synaptic plasticity in the rat dentate gyrus. Neurosci. Lett. 30, 83-86.

61. McGaugh J. L., Cahill L., and Roozendaal B. (1996) Involvement of the amygdala in memory storage: interaction with other brain systems. PNAS 26, 13,508-13,514.

62. Roozendaal B. and McGaugh J. L. (1996b) The memory-modulatory effects of glucocorticoids depend on an intact stria terminalis. Brain Res. 19, 243-250.

63. Packard M. G., Williams C. L., Cahill L., and McGaugh J. L. (1995) The anatomy of a memory modulatory system: from periphery to brain, in Neurobehavioral Plasticity: Learning, Development, and Response to Brain Insults (Speer N. E., Speer L., and Woodruff M., eds.), Erlbaum, Mahwah, NJ, pp. 149-184.

64. Packard M. G., Cahill L., and McGaugh J. L. (1994) Amygdala modulation of hippocampaldependent and caudate nucleus-dependent memory processes. Proc. Natl. Acad. Sci. USA 30, 8477-8481.

65. Roozendaal B. and McGaugh J. L. (1997) Basolateral amygdala lesions block the memory-enhancing effect of glucocorticoid administration in the dorsal hippocampus of rats. Eur. J. Neurosci. 9, 76-83.

66. Roozendaal B., Portillo-Marquez G., and McGaugh J. L. (1996) Basolateral amygdala lesions block glucocorticoid-induced modulation of memory for spatial learning. Behav. Neurosci. 110, 1074-1083. 
67. Roozendaal B., Sapolsky R. M., and McGaugh J. L. (1998) Basolateral amygdala lesions block the disruptive effects of long-term adrenalectomy on spatial memory. Neuroscience $\mathbf{8 4}, 453-465$.

68. Cahill L., Weinberger N. M., Roozendaal B., and McGaugh J. L. (1999) Is the amygdala a locus of "conditioned fear"? Some questions and caveats. Neuron 23, 227-228.

69. Fanselow M. S. and LeDoux J. E. (1999) Why we think plasticity underlying Pavlovian fear conditioning occurs in the basolateral amygdala. Neuron 23, 229-232.

70. Diamond D. M., Puls J. M., Park C. R., and Rose M. G. (2000) Differential effects of stress on hippocampal and amygdaloid LTP: insight into the neurobiology of traumatic memories, in Neuronal Mechanisms of Memory Formation: Concepts of Long-Term Potentiation and Beyond (Holscher C., ed.), Cambridge University Press, Cambridge, UK, pp. 379-404.

71. Lee Y., Walker D., and Davis M. (1996) Lack of a temporal gradient of retrograde amnesia following NMDA-induced lesions of the basolateral amygdala assessed with the fear-potentiated startle paradigm. Behav. Neurosci. 110, 836-839.

72. Maren S., Aharonov G., and Fanselow M. S. (1996) Retrograde abolition of conditional fear after excitotoxic lesions in the basolateral amygdala of rats: absence of a temporal gradient. Behav. Neurosci. 110, 718-726.

73. Wilensky A. E., Schafe G. E., and LeDoux J. E. (1999) Functional inactivation of the amygdala before but not after auditory fear conditioning prevents memory formation. J. Neurosci. 15, RC 48.

74. Liang K. C., McGaugh J. L., Martinez J. L. Jr., Jensen R. A., Vasquez B. J., and Messing R. B. (1982) Post-training amygdaloid lesions impair retention of an inhibitory avoidance response. Behav. Brain Res. 4, 237-249.

75. Parent M. B., Quirarte G. L., Cahill L., and McGaugh J. L. (1995) Spared retention of inhibitory avoidance learning after posttraining amygdala lesions. Behav. Neurosci. 109, 803-807.

76. Clugnet M. C. and LeDoux J. E. (1990) Synaptic plasticity in fear conditioning circuits: induction of LTP in the lateral nucleus of the amygdala by stimulation of the medial geniculate body. J. Neurosci. 10, 2818-2824.

77. Maren S. and Fanselow M. S. (1995) Synaptic plasticity in the basolateral amygdala induced by hippocampal stimulation in vivo. J. Neurosci. 15, 7584-7564.

78. Yaniv D. and Richter-Levin G. (2000) LTP in the rat basal amygdala induced by perirhinal cortex stimulation in vivo. Neuroreport 28, 525-530.

79. Rogan M. T. and LeDoux J. E. (1996) Emotion: systems, cells, synaptic plasticity. Cell 17, 469-75.

80. McKernan M. G. and Shinnick-Gallagher P. (1997) Fear conditioning induces a lasting potentiation of synaptic currents in vitro. Nature 390, 607-611.

81. Rogan M. T., Staubli U. V., and LeDoux J. E. (1997) Fear conditioning induces associative long-term potentiation in the amygdala. Nature 1, 604-607.

82. Akirav I., Sandi C., and Richter-Levin G. (2001). Hippocampus and amygdala involvement in spatial learning as a function of stress. Submitted.

83. Canteras N. S. and Swanson L. W. (1992) Projections of the ventral subiculum to the amygdala, septum, and hypothalamus: a PHAL anterograde tract-tracing study in the rat. J. Comp. Neurol. 8, 180-194.

84. Ottersen O. P. (1982) Connections of the amygdala of the rat. IV: Corticoamygdaloid and intraamygdaloid connections as studied with axonal transport of horseradish peroxidase. $J$. Comp. Neurol. 10, 30-48.

85. Swanson L. W. and Kohler C. (1986) Anatomical evidence for direct projections from the entorhinal area to the entire cortical mantle in the rat. J. Neurosci. 6, 3010-3023.

86. Wyss J. M. (1981) An autoradiographic study of the efferent connections of the entorhinal cortex in the rat. J. Comp. Neurol. 199, 495-512.

87. Mello L. E., Tan A. M., and Finch D. M. (1992a) GABAergic synaptic transmission in projections from the basal forebrain and hippocampal formation to the amygdala: an in vivo iontophoretic study. Brain Res. 31, 41-48.

88. Mello L. E., Tan A. M., and Finch D. M. (1992b) Convergence of projections from the rat hippocampal formation, medial geniculate and basal forebrain onto single amygdaloid neurons: an in vivo extra- and intracellular electrophysiological study. Brain Res. 31, 24-40.

89. Phillips R. G. and LeDoux J. E. (1992) Differential contribution of amygdala and hippocampus to cued and contextual fear conditioning. Behav Neurosci. 106, 274-285. 
90. Morgan M. A. and LeDoux J. E. (1995) Differential contribution of dorsal and ventral medial prefrontal cortex to the acquisition and extinction of conditioned fear in rats. Behav. Neurosci. 109, 681-688.

91. Morgan M. A. and LeDoux J. E. (1999) Contribution of ventrolateral prefrontal cortex to the acquisition and extinction of conditioned fear in rats. Neurobiol. Learn. Mem. 72, 244-251.

92. Doyere V., Burette F., Negro C. R., and Laroche S. (1993) Long-term potentiation of hippocampal afferents and efferents to prefrontal cortex: implications for associative learning. Neuropsychologia 31, 1031-1053.
93. Jay T. M., Burette F., and Laroche S. (1995) NMDA receptor-dependent long-term potentiation in the hippocampal afferent fiber system to the prefrontal cortex in the rat. Eur. J. Neurosci. 1, 247-250.

94. Jay T. M., Burette F., and Laroche S. (1996) Plasticity of the hippocampal-prefrontal cortex synapses. J. Physiol. Paris 90, 361-366.

95. Laroche S., Jay T. M., and Thierry A. M. (1990) Long-term potentiation in the prefrontal cortex following stimulation of the hippocampal CA1/subicular region. Neurosci. Lett. 3, 184-190. 